\title{
Contralateral Vascularized Lymph Node Transfer: An Optimized Mouse Model
}

\author{
Giulia Virginia Vottero, MD ${ }^{1, *(1)}$ Florent Morfoisse, $\mathrm{PhD}^{2, *}$ Tania Durré, PhD ${ }^{2}$ Silvia Blacher, $\mathrm{PhD}^{2}$ \\ Guillaume Becker, $\mathrm{PhD}^{3}$ Mohamed A. Bahri, PhD ${ }^{3}$ Alain Plenevaux, PhD ${ }^{3}$ Agnès Noel, $\mathrm{PhD}^{2}$ \\ Jean Luc Nizet, MD, $\mathrm{PhD}^{1}$
}

${ }^{1}$ Department of Plastic and Maxillofacial Surgery, University Hospital of Liège, Liège, Belgium

${ }^{2}$ GIGA-Cancer Department, Laboratory of Biology of Tumor and Development, GIGA-Cancer, University of Liège, Liège, Belgium

${ }^{3}$ GIGA-Cyclotron Research Centre/In Vivo Imaging, University of Liège, Liège, Belgium

J Reconstr Microsurg Open 2019;4:e83-e91.
Address for correspondence Giulia Virginia Vottero, MD, Department of Plastic and Maxillofacial Surgery, University Hospital of Liège, Domaine du Sart Tilman, B.45, Liège, 4000, Belgium (e-mail: giulia_vottero@yahoo.it).

\begin{abstract}
Keywords

- lymphedema

- mouse model

- vascularized lymph node transfer

Background Vascularized lymph node transfer (VLNT) is one of the surgical options in the treatment of lymphedema, but its mechanism of action has not yet been firmly clarified. In the VLNT mouse models described so far, the lymph node flap is performed between two different sites in the same lymphedematous paw. In this study, we describe an optimized VLNT mouse model using the contralateral paw as donor site, thus removing the bias of transferring a lymph node already damaged by irradiation and/or surgery required to induce lymphedema.

Methods A lymphedema was induced on the left posterior paw in four experimental groups of mice $(n=8)$. Two weeks later, group 1 was the sham one, group 2 underwent a VLNT from the right inguinal region to the left, in group 3 a vascular endothelial growth factor (VEGF)-C sponge was placed alone in the left inguinal region, and in group 4 a VEGF-C sponge was associated to the VLNT. The 32 mice were followed during 3 months. Outcomes included paws volume, skin quality, inflammation in the lymphedematous tissue, and lymphatic network density and function.

Results Group 4 displayed significantly higher $(p<0.05)$ lymphedema regression compared with the other three groups.

Conclusions This optimized mouse model of VLNT shows to be handy and effective. It could be exploited to perform further experimental studies about the influence of VLNT on lymphedema. Moreover, the local association between VLNT and biological compounds in this model allows it to be a good preclinical model to identify new potential drugs in lymphedema.
\end{abstract}

Lymphedema is a progressive, usually unrelenting, and variably painful swelling of the limbs and/or genitalia resulting from lymphatic system insufficiency and disturbed lymphatic transport. In developed countries, malignant neoplasms and their

These authors contributed equally to this manuscript. therapies are the most common cause of lymphedema; breast cancer-related lymphedema of the arm is the most prevalent form. Nodal dissections and/or radiation therapy for gynecological, genitourinary, and head/neck malignancies have been implicated in the development of lymphedema. ${ }^{1}$ Lymphedema has been treated conservatively or surgically. ${ }^{2,3}$ Vascularized received

April 24, 2019

accepted after revision

September 29, 2019
DOI https://doi.org/

10.1055/s-0039-3400243. ISSN 2377-0813.
Copyright $\odot 2019$ by Thieme Medical Publishers, Inc., 333 Seventh Avenue, New York, NY 10001, USA. Tel: +1(212) 584-4662.
License terms

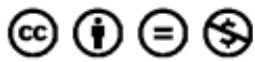


lymph node transfer(VLNT) showed clinical promising results, ${ }^{4}$ but its mechanism of action has not yet been firmly clarified. To better understand the lymphedema pathology and therapeutic options, many animal models have been set up. ${ }^{5}$ Mice models of limb lymphedema obtained by a combination of surgical lymphatic flow interruption and irradiation can reproduce the human pathology with good approximation. Because of their cost efficiency, mice models are constantly under revision and improvement. ${ }^{6}$ One of the major limitations in performing a VLNT hindlimb mouse model is the small size of the vascular pedicle of the lymph node flap, which represents a technical limitation to the achievement of a free vascular anastomosis, in contrast to what has been recently performed on a rat $\operatorname{model}^{7}$ and what is routinely performed in humans. Until now, to overcome this limitation, VLNT in mice was realized as a pedicle flap harvesting VLNT from inguinal (also called "abdominal") to popliteal in the same paw. ${ }^{8-11}$ We believe that the ipsilateral lymph node transfer can represent a limitation in the performance of a VLNT mouse model for two reasons: (1) in case of a VLNT in a lymphedema paw obtained by irradiation on the limb, reasonably the lymph node to transfer will be damaged, as the irradiation decreases lymphatic reserve by causing depletion of lymphatic vessels and lymphatic endothelial cells as well as promoting soft tissue fibrosis. ${ }^{12}(2)$ Given that in mice the inguinal and popliteal lymph nodes both drain lymph from the hind leg, ${ }^{13}$ removing the popliteal lymph node and harvesting the ipsilateral inguinal one to transfer it in the popliteal region additionally impairs the lymphatic drainage of the paw, which is not ideal for a VLNT model. For this reason and to preserve the lymphedematous side from any supplementary lymphatic disruption, we used the contralateral paw as donor site in alternative from other studies. Because of the smaller size of the circumflex superficial iliac artery in mice and to get a VLNT animal model that is technically easy to perform, the lymph node flap has been pedicle, in contrast with free microsurgical transfer recently described in rats. ${ }^{7}$ In line with the literature, ${ }^{5}$ we decided to apply the contralateral VLNT to the acquired and chronic hindlimb mouse model described by Oashi et al in 2012. ${ }^{14}$ As vascular endothelial growth factor (VEGF)-C has been shown to improve lymphangiogenesis in experimental studies, ${ }^{15,16}$ we embedded it in gelatin sponges and locally added it to the VLNT to quantify its lymphangiogenic influence. According with the long-lasting lymphedema mice model described by Oashi et al, ${ }^{14}$ the lymphedema is expected to decrease in the left hindlimb progressively and spontaneously 20 days after the first surgery. At day 63, the paw circumference increases again until day 87 , after that the volume finally decreases to become similar to the preoperative circumference. In consequence, we decided to follow the mice during 90 days after the lymphedema surgery, to be sure that any lymphedema volume regression observed will not be the consequence of spontaneous recovery. We used as control both the right paw of each group and the right (untouched) paws of the sham group. Our findings provide a more accurate and easy-to-handle VLNT mouse model, applying the combination of VLNT and a biological compound (VEGF-C in this case) locally released using gelatin sponges. Interestingly, these sponges are suitable to encapsulate a wide

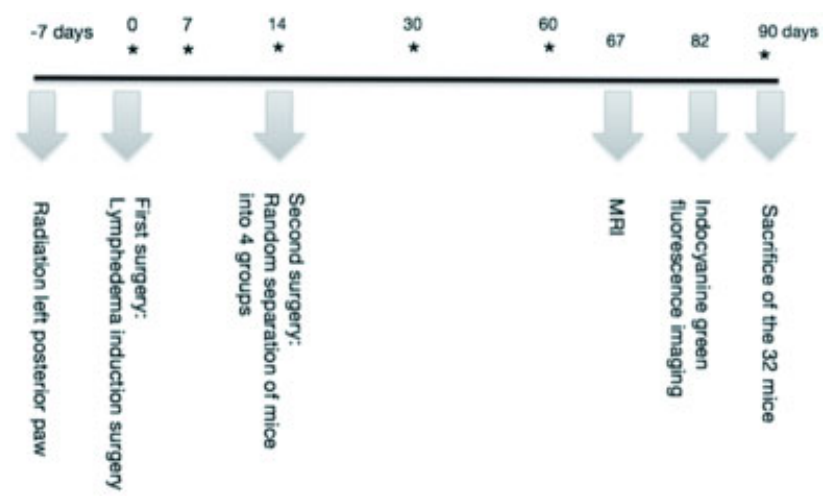

Fig. 1 Summary of the principal steps and timing of the study. ${ }^{*}$ Paws diameter measurement.

range of molecules, so this technique can be beneficial to test the therapeutic potential of exogenous factors associated with VLNT and its consequence on lymphedema.

\section{Methods}

All experiments were conducted in accordance with the national and institutional guidelines for the care and use of animals in research. This study was approved by the Ethics and Scientific Integrity Council (C.E.I.S.) of the University of Liège, Liège, Belgium (Dossier n. 18-2074). The different steps are summarized in - Fig. $\mathbf{1}$.

\section{Lymphedema Mouse Model}

Thirty-two, 8-week-old C57BL/6 mice (Charles River Laboratories, The Netherlands) were used in this study. Mice were kept at a temperature of $23^{\circ} \mathrm{C}$ with $50 \%$ humidity and 12:12-hour lightdark cycles, with free access to water and standard feed. During irradiation, the animals were anesthetized with isoflurane (IsoFlo, Zoetis, Belgium) and irradiated by a single dose of 30 Gy focused on the left inguinal region (X-RAD SmART, GE Healthcare, Belgium). One week after irradiation, mice $(n=32)$ underwent the surgical induction of lymphedema on the left posterior paw. During the surgery, the animals were anesthetized with isoflurane and the whole procedure was performed under a laminar airflow hood (Clean Air Techniek B.V., The Netherlands). A dose of $5 \mu \mathrm{L}$ of $2 \%$ patent blue dye (Sigma-Aldrich) was injected between the footpads of the left inferior paw. The lymphedema was induced by three surgical steps: circumferential skin incision at the left inguinal region, excision of the inguinal and popliteal nodes, and ligature of lymphatic collectors beside the ischiatic vein at least at three different levels by stitches of 10/0 nylon (Monosoft, COVIDIEN; Medtronic, Switzerland) under a Leica M80 manual microscope (Leica Microsystems, Germany) at $10 \times$ magnification. After the procedure, the circumferential skin incision was sutured by absorbable separated stitches of 6/0 nylon (Polysorb, COVIDIEN; Medtronic, Switzerland).

\section{Grouping of the Experimental Animals}

Two weeks later, the second surgical procedure was performed (see next part). At this moment, the 32 mice were separated into four groups of eight. 
Group 1 (Sham): $n=8$ : No VLNT and no VEGF-C sponge in the left inguinal region.

Group 2 (VLNT): $n=8$ : VLNT and no VEGF-C sponge in the left inguinal region.

Group 3 (VEGF-C): $n=8$ : No VLNT and VEGF-C sponge in the left inguinal region.

Group 4 (VLNT + VEGF-C): $n=8$ : VLNT and VEGF-C sponge positioned under the transferred lymph node in the left inguinal region.

The left posterior paw of group 1 was used as control for the lymphedema evolution and the right inferior paw of the same group served as control compared with the nonlymphedema paws of the other groups, as it did not suffer any radiation nor lymph node excision.

\section{VLNT and VEGF-C Sponge Placement}

During the performance of the second surgery, 16 gelatin sponges of Gelfoam (Pfizer, Belgium) measuring approximately $3 \mathrm{~mm}^{3}$ were incubated with a solution of human VEGF-C (R\&D Systems) at a concentration of $1 \mu \mathrm{g} / \mathrm{sponge}$. After 1 hour of incubation, the sponges were immediately implanted in the left inguinal region. ${ }^{17}$

The second surgical procedure was performed under general anesthesia by isoflurane and surgical loop magnification of $3.5 \times$ (Univet S.r.l., Italy). The abdominal skin was incised in a V-shaped flap to expose both inguinal regions (-Fig. 2) in all the 32 mice. In groups 2 and 4, the contralateral VLNT was performed as follows: the right inguinal lymph node was dissected pedicle based on the superficial iliac circumflex artery and vein and the pedicle was dissected up to its origin at the femoral vessels (-Fig. 3). The lymph node was identified but not completely dissected from the adipose tissue to preserve the lymphatic-vascular connections. Then, the lymph node was turned 180 degrees using the origin of the vascular pedicle as the pivot point, and fixed on the left inguinal region by three absorbable stitches of $6 / 0$ nylon (-Fig. 4). In group 4, the VEGF-C soaked sponge was placed in

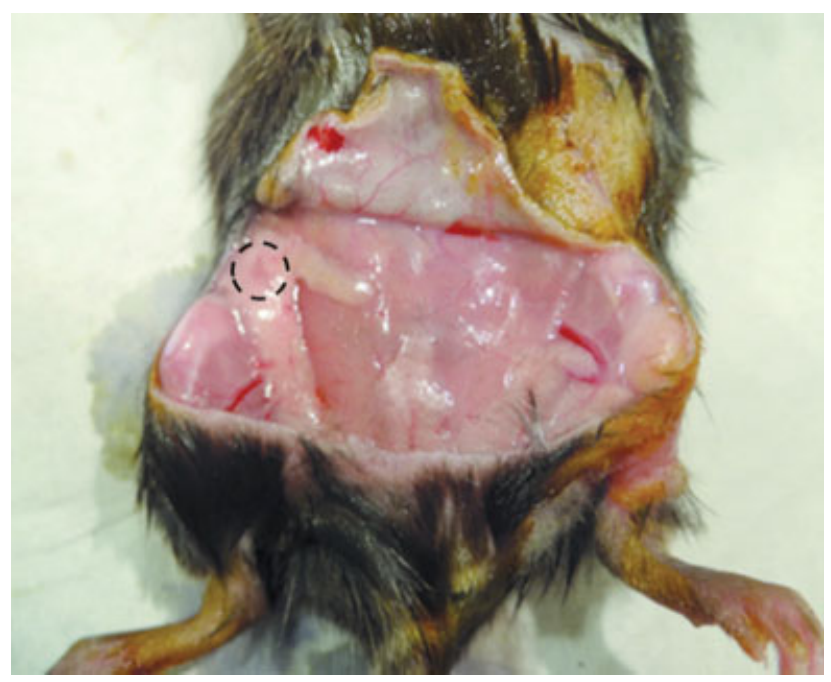

Fig. 2 Exposure and isolation of the right inguinal lymph node (circle) by a V-shaped abdominal skin incision to access to both inguinal regions.

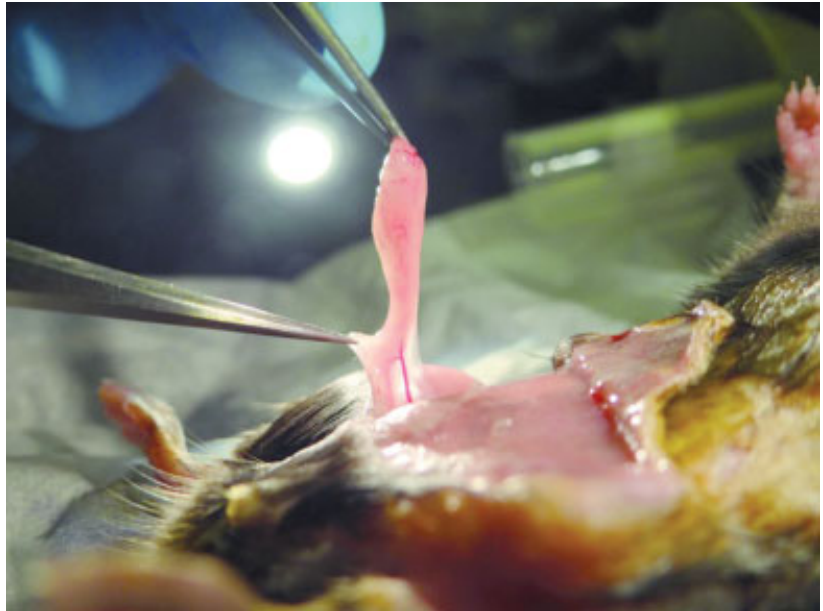

Fig. 3 Dissection of the right inguinal lymph node and its vascular pedicle from its origin.

the inguinal cavity under the transferred lymph node before suturing it to the connective surrounding inguinal tissue, so the transferred tissue weight assured the contact between the lymphatic tissue and the growth factor preventing the sponge from moving. In group 3, the sponge with VEGF-C was fixed in a pouch of connective tissue firmly folded by three stitches of absorbable 6/0 nylon (-Fig. 5). The abdominal skin flap was closed by a running suture of absorbable $6 / 0$ nylon in all 32 mice.

\section{Measurement of Hindlimb Paw Volume and Skin Breakdown Assessment}

At day $0,7,14,30,60$, and 90 (day of sacrifice), the same observer measured by the help of a caliper the left and right posterior paw radius at the groin $(R)$ and at the knee $(r)$ and the distance between these two points $(h)(-$ Fig. 1$)$. The paw

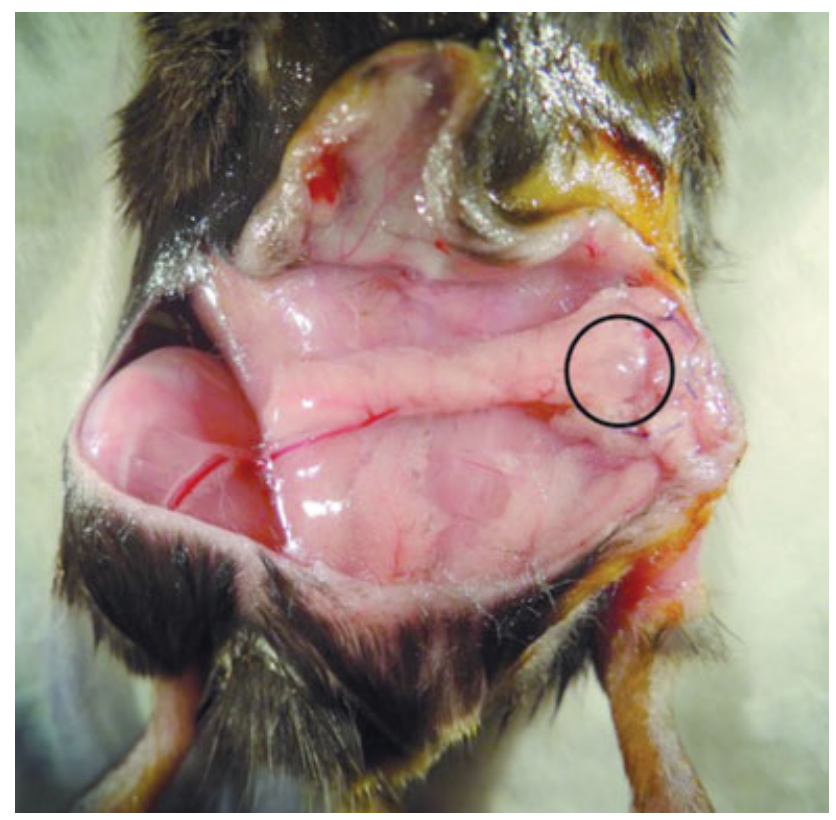

Fig. 4 The vascularized lymph node transfer (VLNT) is fixed in the left inguinal region. 


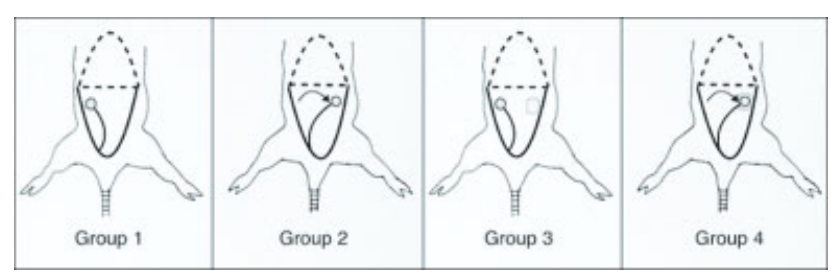

Fig. 5 The four experimental groups, from left to the right: group 1 (Sham), group 2 (VLNT), group 3 (VEGF-C), and group 4 (VLNT + VEGF-C). Circle: right inguinal lymph node. Rectangle: VEFG-C sponge placed in the left groin, under the lymph node.

volume $(V)$ was calculated using the truncated cone formula:

$$
V=h / 3 \pi\left(R^{2}+R r+r^{2}\right)
$$

Left hindlimbs were scrutinized weekly by the same observer for any signs of skin breakdown and/or wound oozing. The skin aspect was qualified by a visual assessment scale describing four different grades: Grade $0=$ no ulcer, Grade $1=$ isolated ulcer without oozing, Grade $2=$ isolated ulcer with oozing, and Grade $3=$ ulcers and oozing on the whole limb.

\section{Lymphedema and Skeletal Muscle Volume Assessment by Magnetic Resonance Imaging}

At day 85, the mice underwent an in vivo magnetic resonance imaging (MRI) scan to assess the lymphedema volume. For each mouse, a T2 anatomical images was acquired on a $9.4 \mathrm{~T}$ MRI DirectDrive VNMRS horizontal bore system with a shielded gradient system (Agilent Technologies, Palo Alto, CA) using a 40-mm inner diameter volumetric coil (Agilent Technologies). During the imaging session, mice were under general anesthesia by isoflurane in a mixture of $30 \%$ of $\mathrm{O}_{2}$ in air, placed in a prone position in a dedicated animal holder equipped with an air warming system. The mice were monitored during the whole session (respiratory rate and temperature). Just before loading the acquisition, the mice received an intraperitoneal bolus injection of $100 \mu \mathrm{L}$ of $0.5 \mathrm{mmol} / \mathrm{mL}$ Gadovist (Bayer, Germany). A fast spin echo multislice MRI sequence was used with the following parameters: $\mathrm{TR}=128 \mathrm{~ms}, \mathrm{TE}=6 \mathrm{~ms}$, field of view $(\mathrm{FOV})=35 \times 35 \mathrm{~mm}^{2}$, matrix $192 \times 192$ leading to an in-plane voxel size of $0.182 \mathrm{~mm}$. Fifteen coronal slices covering the two hindlimbs were acquired with a thickness of $1 \mathrm{~mm}$.

Structural MRI images were used to assess the skeletal muscle volume as well as the total volume (fat, bone, muscle) of each hindlimb (lymphedema and contralateral). For this aim, a semiautomated segmentation procedure was employed using the PMOD 3.6 software (PMOD Technologies, Zurich, Switzerland; RRID:SCR_016547). Structural MRI image was manually segmented to create two images containing the lymphedema and contralateral hindlimbs. Based on the mean signal intensity (threshold: 450-900), extracted on a spherical region of interest placed on the muscle of both hindlimbs, binary masks of the hindlimbs muscle were extracted using automated segmentation method implemented in PMOD. Knowing the voxel size and the number of voxels in the obtained masks, the muscle volume for each hindlimb was then calculated.

\section{Indocyanine Green Fluorescence Imaging}

Mice were anesthetized with $2 \%$ isoflurane and then positioned inside an IVIS 200 Vivo Vision (Xenogen; Caliper Life Sciences) on their dorsal side, and precontrast injection images were taken to establish background signal intensities at tissues of interest. The imaging parameters were as follows: $\lambda \mathrm{ex}=745 \mathrm{~nm}, \lambda e \mathrm{~m}=840 \mathrm{~nm}$, exposure time $=1$ second, $\mathrm{f} / \mathrm{stop}=2$, small binning, $\mathrm{FOV}=6.6 \times 6.6 \mathrm{~cm}^{2}$. Four microliters of indocyanine green dye (Verdye $0.1 \mathrm{mg} / \mathrm{mL}$, Diagnostic Green, Germany) were injected into both paws between the cushions. Images were acquired immediately after injection ( $T=5$ minutes) and at different time points postinjection (1, 3 , 5 , and 7 hours). For image analysis, Living Image software (Caliper Life Sciences) was used. Regions of interest (ROIs) were placed on the injected paw. Average signal intensity values were recorded for each ROI and plotted versus time in GraphPad Prism.

\section{Lymphatic Vessel Immunostaining}

At day 90, the 32 mice were euthanized. The skin sections were harvested distal to the inguinal wound on the medial tight, to avoid the sampling of scar tissue. The tissue was fixed immediately with $4 \%$ paraformaldehyde and embedded in paraffin. Sections were blocked for 20 minutes in AnimalFree Blocking Solution (15019 L; Cell Signaling Technology, The Netherlands) and incubated for 2 hours at room temperature with either F4/80 (1/250; 70076, Cell Signaling Technology) or LYVE-1 (1/ 200; AF2125, R\&D Systems, United Kingdom) antibodies or incubated overnight at $4^{\circ} \mathrm{C}$ with CD45 antibody (1/200; 70257, Cell Signaling Technology). After washing in phosphate-buffered saline, slides were incubated with VectaFluor Excel Amplified DyLight 488 (DK-1488, Vector Laboratories, United Kingdom). Staining was quantified with a computerized method.

\section{Computerized Quantification Method}

Images from whole immunolabeled tissues were captured with the fully automated digital microscopy system (Hamamatsu Photonics, Belgium) at a resolution of $0.46 \mu \mathrm{m} /$ pixel (20× magnitude). Original image analysis algorithm was implemented to automatically perform image processing and measurements, by using the image analysis toolbox of MATLAB (R2018a) software (MathWorks). By this process, the lymphatic vessel, LYVE-1, F4/80, and CD45 stainings ${ }^{18}$ densities, defined as the number of pixels belonging from staining cells (staining area) divided by number of pixels belonging from the whole tissue (tissue area), were automatically determined.

\section{Statistical Analysis}

Results were analyzed with GraphPad Prism 5.0 and were expressed as means \pm standard error of the mean. The $p$-values were obtained using the Mann-Whitney test, two-tailed (not assuming Gaussian distributions); $p<0.05$ was considered as statistically significant and was represented on graphs as 


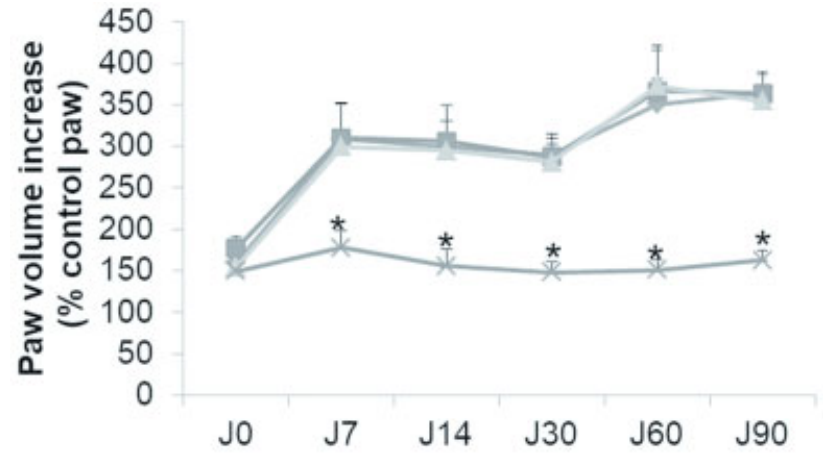

Fig. 6 Left limb volume evolution during the 90 days' follow-up. Results are expressed as the mean volume increase (\% of control) of the operated hindlimbs compared with the control one in each experimental group.

follows: ${ }^{*} p<0.05,{ }^{* *} p<0.01$, and ${ }^{* * *} p<0.001$. The experiments were not randomized. No blinding was done in the analysis and quantifications.

\section{Results}

\section{Paw Volume and Skin Breakdown Analysis}

Time course analysis of lymphedema development revealed that the left hind paw volume of group 4 was significantly lower than in the other experimental groups at 7, 14, 30, 60, and 90 days after surgery $(p<0.05)$ ( - Fig. 6 ). The skin breakdown was significantly minor in group 4 compared with the other groups $(p<0.05)$ ( - Fig. 7$)$.

\section{Indocyanine Green Fluorescence Imaging}

To analyze the fluid clearance in the limb, we injected indocyanine green between both control and lymphedematous cushion paws and measured the intensity of the fluorescent signal at several time points. We observed a strong accumulation of indocyanine into the lymphedematous paws 1 hour after the injection followed by a progressive clearance as revealed by the slow decrease of the fluorescent signal. It is worth noting that in group 4, the fluorescent signal measured 1 hour after the injection was much weaker than the one of the three other groups meaning that indoc-

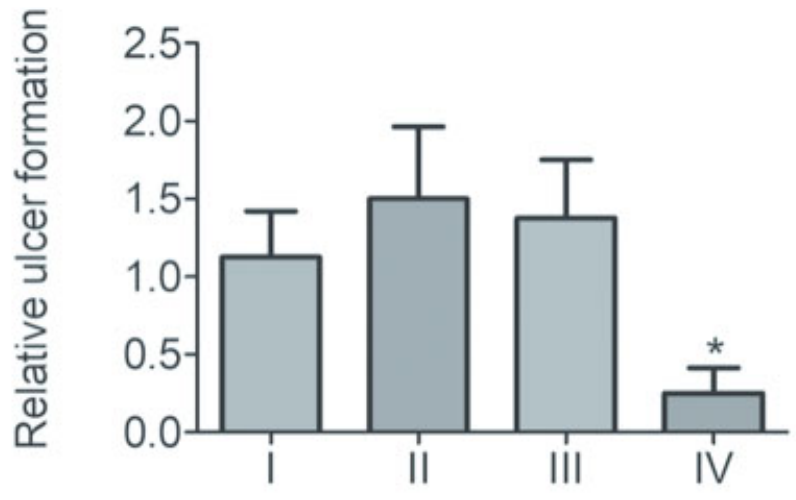

Fig. 7 Skin breakdown in the four experimental groups at day 90 . Group 4 (IV) showed minimal skin breakdown in comparison to the other three groups $(p<0.005)$.

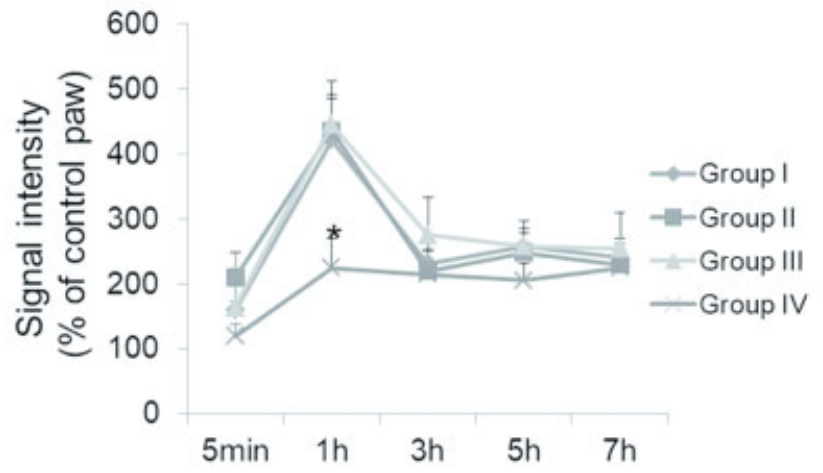

Fig. 8 Evolution of the signal intensity in the left inferior paw at different times after indocyanine green injection in the four groups. Graphed over time is the mean fluorescence intensity increase in the operated hindlimbs compared with the control one. Group 4 showed a significant $(p<0.05)$ lower intensity of the signal 1 hour after injection, in accord with a better lymphatic reabsorbtion.

yanine accumulated to a lesser extent. This result reveals that the drainage was more efficient in mice that received both VLNT and VEGF-C treatment. Although we cannot differentiate the contribution of blood or lymphatic vessels in the drainage in this experiment, it has been well described that lymphatic vessels and particularly lymphatic capillaries are more implicated in tissue fluid drainage than blood vessels. ${ }^{19}$ This experiment thus strongly suggest that lymphatic functionality was improved in group 4 compared with the others (-Figs. 8 and 10).

\section{Density of Lymphatic Vessels with LYVE-1 Immunoreactivity}

Skin lymphatic vasculature was assessed by LYVE-1 immunostaining. We observed a significant decrease of lymphatic coverage in the skin of the lymphedematous limbs compared with their control counterparts in groups 1, 2, and 3. Interestingly, in group 4 the lymphatic density was the same in both control and lymphedematous skin. Moreover, the
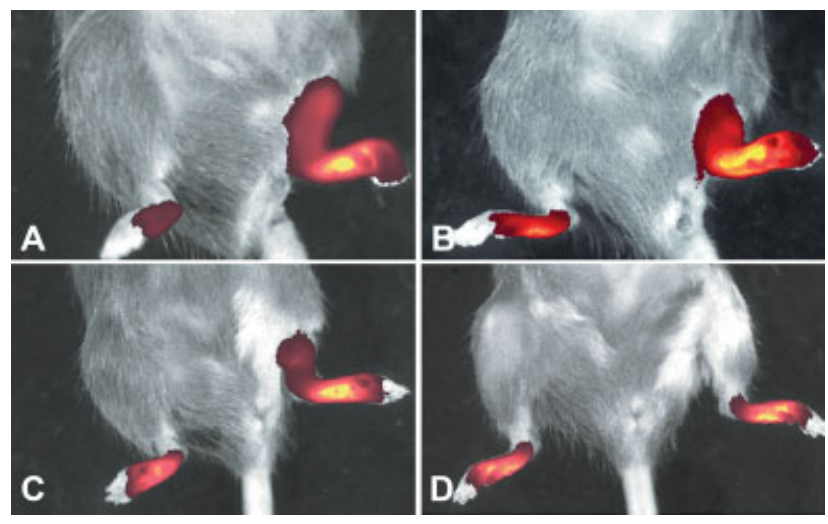

Fig. 10 Analysis of lymphatic clearance by indocyanine green injection at day 82. Group 1 (A), group 2 (B), group 3 (C), and group 4 (D) indocyanine green clearance at 1 hour after injection. Group 4 shows a lower infrared signal of theoperatedpawconfirminga better lymphatic clearance than the other three groups $(p<0.05)$ at 1 hour after injection. 


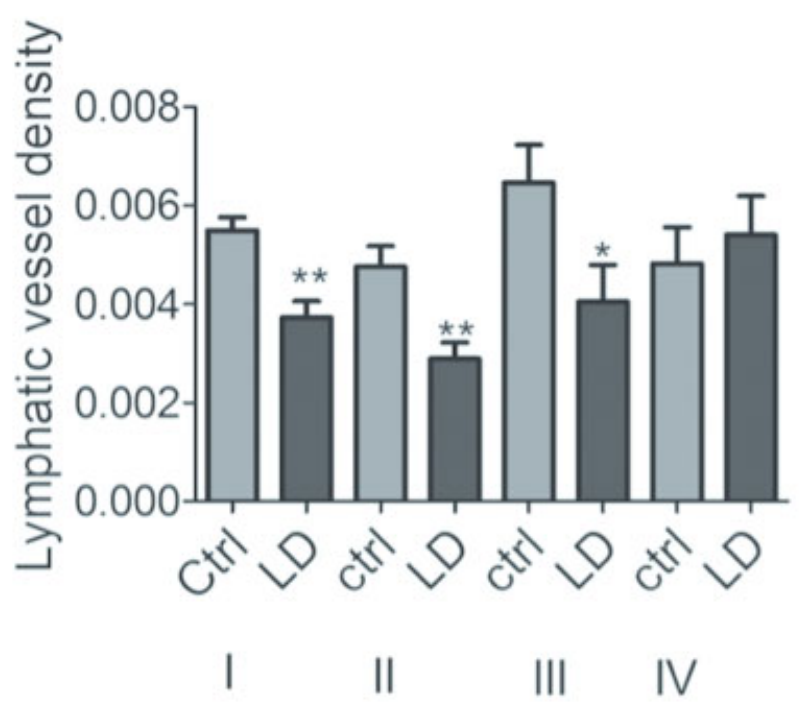

Fig. 9 Lymphatic vessel density in the four groups assessed by immunohistochemical staining (LYVE-1). Group 4 shows the highest lymphatic vessel density in comparison to groups 1 and $2(p<0.001)$ and group $3(p<0.05)$.

lymphatic density in lymphedematous skin in group 4 tended to be higher than the one of the three others groups suggesting that the reduction of edema development observed in group 4 might be due to a better-preserved lymphatic system (-Figs. 9 and 11).

\section{Analysis of $F 4 / 80$ and CD45 Expression}

To quantify the inflammation, which is a chronic finding in lymphedema, ${ }^{20}$ we analyzed the recruitment of immune cells in the skin of control and lymphedematous limbs using both F4/80 (a specific marker of macrophages) and CD45 (global marker of inflammatory cells) immunostainings. We observed that lymphedematous skin in groups 1, 2, and 3 displayed higher content of both F4/80 ( - Fig. 12) and CD45 (-Fig. 13) positive cells compared with their control counterparts revealing a higher inflammatory response. At the contrary, lymphedema in group 4 exhibited the same levels of F4/80 (-Fig. 14) and CD45 (-Fig. 15) positive cells as control skins revealing that the inflammation resolved at the moment of sacrifice.

\section{MRI Analysis of the Tissue's Component}

To evaluate more precisely the lymphedema volume, an MRI approach was used. As observed with the first macroscopic measurement of the paw, the lymphedematous limb in group 4 is significantly smaller compared with the three others groups (-Fig. 16). Interestingly, we revealed that, compared with their control counterparts, lymphedematous limbs in groups 1, 2, and 3 exhibit a significant decrease of muscle volume. At the opposite, muscle volume of the lymphedematous hindlimb in group 4 was unaffected compared with the control limb (-Figs. 17 and 18). This skeletal muscle response to lymphedema has been already noticed in human, ${ }^{21,22}$ but is poorly investigated on mice models and never been reported in literature.

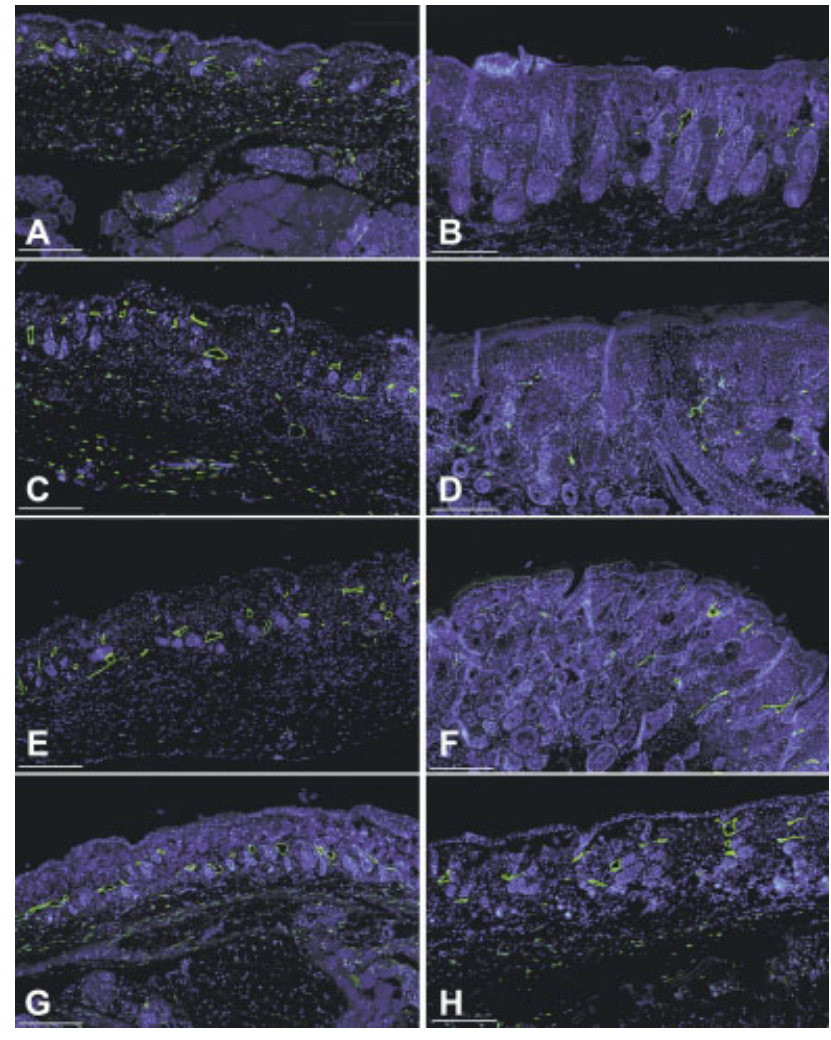

Fig. 11 Identification of lymphatic vessels by LYVE-1 antibody. Images A, C, E, and G show the concentration of lymphatic vessels (green) in the skin of the control (right) paws in groups 1, 2, 3, and 4, respectively. Images $\mathrm{B}, \mathrm{D}, \mathrm{F}$, and $\mathrm{H}$ show the concentration of lymphatic vessels in the skin of the lymphedema (left) paws in groups $1,2,3$, and 4 , respectively. Group 4 shows the highest lymphatic vessels density $(\mathbf{H})$ in comparison to groups 1 (B) and 2 (D) $(p<0.001)$ and group $3(F)(p<0.05)$. The lymphatic vessels density in the lymphedema paw in group $4(\mathrm{H})$ shows no statistical difference compared with its control paw (G) and the control paw of group 1 (healthy paw, A). Scale bar: $200 \mu \mathrm{m}$.

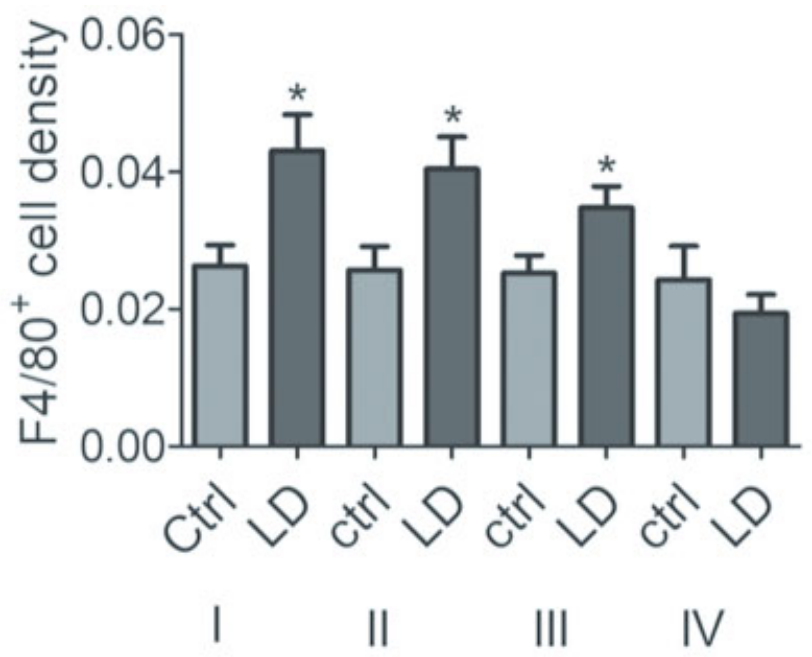

Fig. 12 The $\mathrm{F} 4 / 80^{+}$cell density (inflammation cells) in the four groups. Group 4 showed the lowest $\mathrm{CD} 45^{+}$cell density in comparison to the other three groups $(p<0.05)$. 


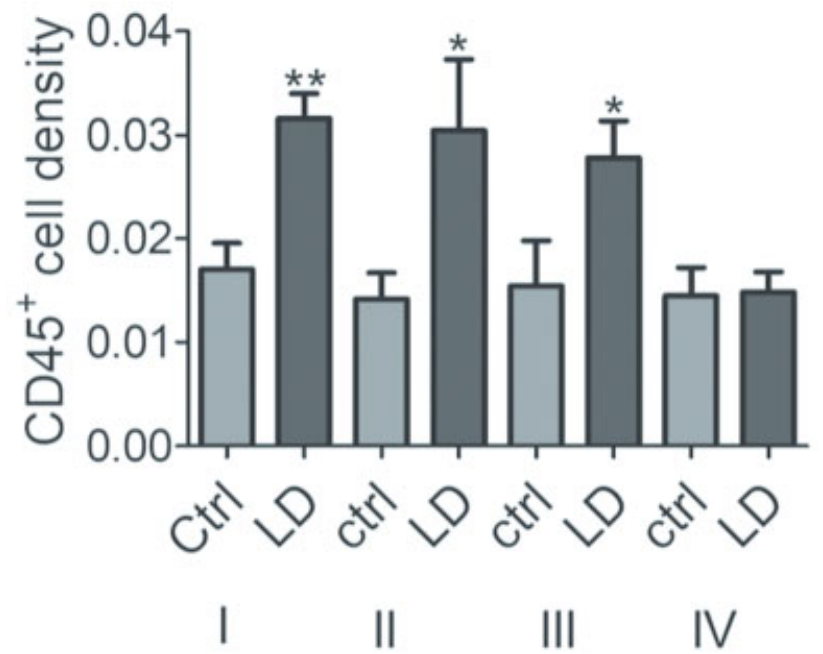

Fig. 13 The $\mathrm{CD} 45^{+}$cell density (inflammation cells) in the four groups. Group 4 showed the lowest $\mathrm{CD} 45^{+}$cell density in comparison to group $1(p<0.001)$ and groups 2 and $3(p<0.05)$.

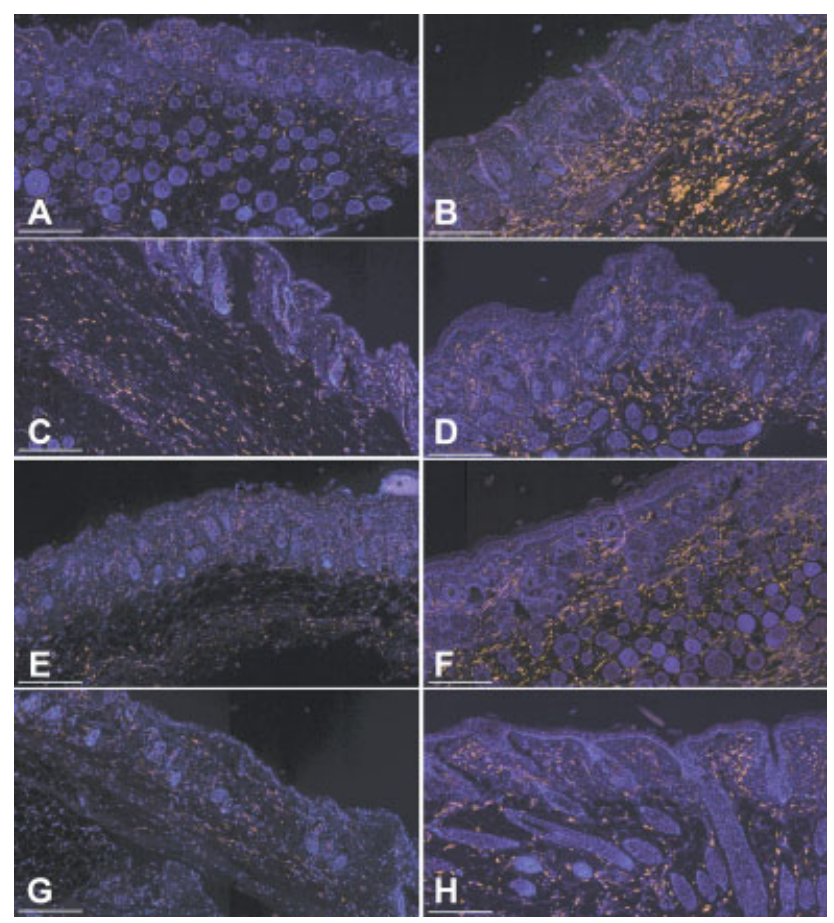

Fig. 14 Identification of $\mathrm{F} 4 / 80^{+}$cells. Images A, C, E, and G show the concentration of $\mathrm{F} 4 / 80^{+}$cells in the skin of the control (right) paws in groups 1, 2, 3, and 4, respectively. Images B, D, F, and $\mathbf{H}$ show the concentration of $\mathrm{F} 4 / 80^{+}$cells in the skin of the lymphedema (left) paws in groups $1,2,3$, and 4 , respectively. The lymphedema paw of group $4(\mathrm{H})$ shows the lowest density of $\mathrm{F} 4 / 80^{+}$cells in comparison to the other three groups (B, D, F) $(p<0.05)$. Scale bar: $200 \mu \mathrm{m}$.

\section{Discussion}

The clinical success of the VLNT depends on the ability of the grafted lymphatic tissue to recreate new and functional lymphatic connections. Indeed, the VLNT outcome relies on the survival of the lymphatic graft, on the quality of the recipient site (after radiation and/or surgery), and on the stage of the patient disease in correlation with the deposition

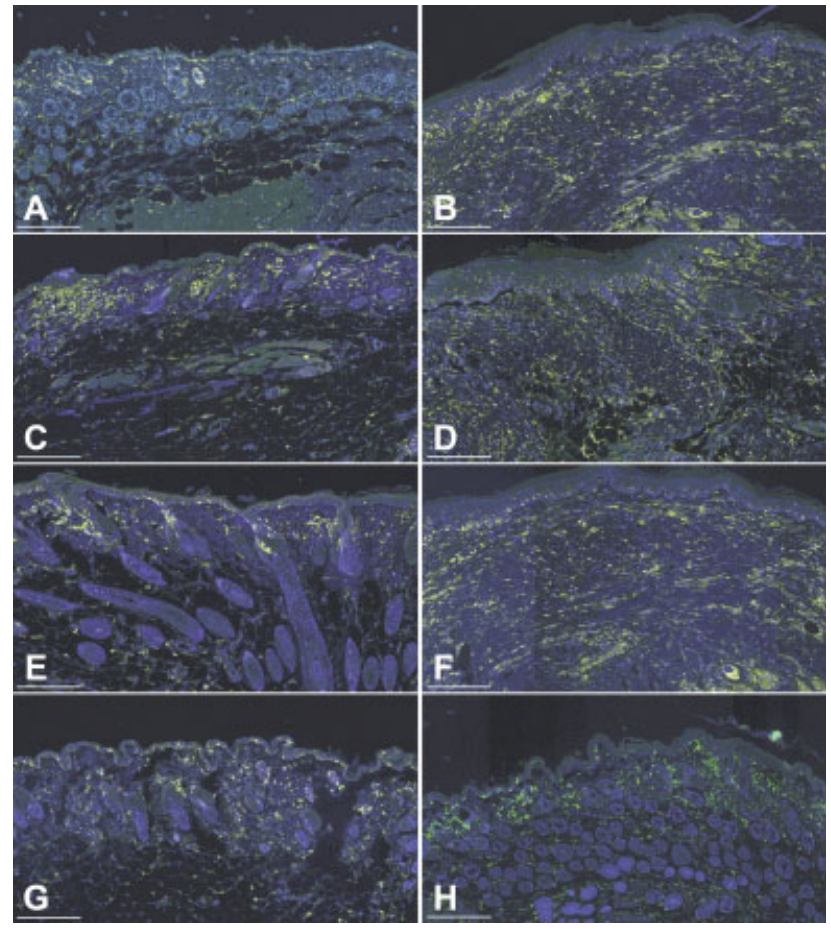

Fig. 15 Identification of $\mathrm{CD}^{4} 5^{+}$cells. Pictures A, C, E, and G show the concentration of $\mathrm{CD} 45^{+}$cells in the skin of the control (right) paws in groups $1,2,3$, and 4 , respectively. Images $\mathbf{B}, \mathbf{D}, \mathbf{F}$, and $\mathbf{H}$ show the concentration of $\mathrm{CD} 45^{+}$cells in the skin of the lymphedema (left) paws in groups $1,2,3$, and 4 , respectively. The lymphedema paw of group $4(\mathrm{H})$ shows the lowest density of $\mathrm{CD} 45^{+}$cells in comparison to the other three groups (B, D, F) $(p<0.05)$. Scale bar: $200 \mu \mathrm{m}$.

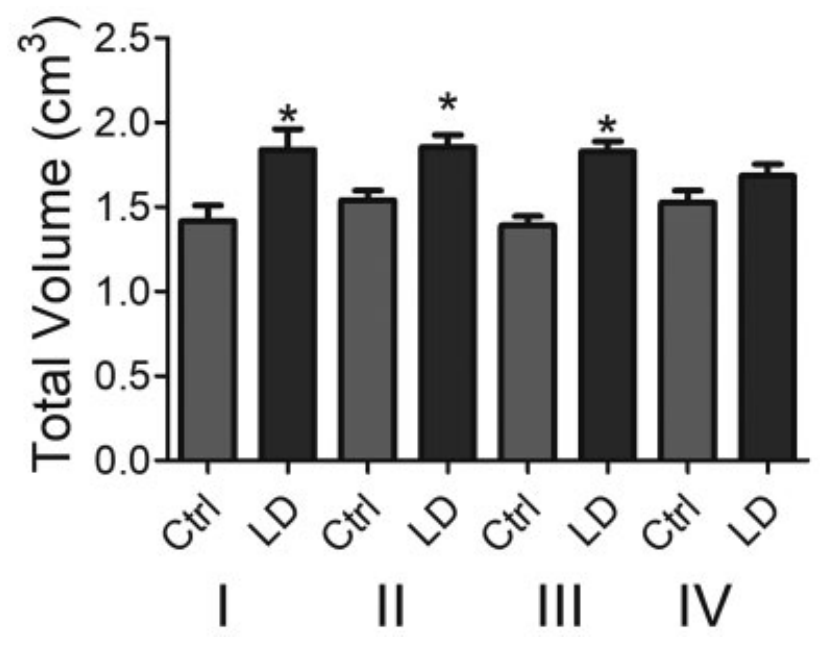

Fig. 16 Paw volume evaluated by magnetic resonance imaging (MRI) measurement at day 67 after lymphedema induction. Group 4 showed no significant total volume increase of the operated limbs, compared with the control one.

of fibrosis and adipose tissue. ${ }^{23}$ As many studies refine lymphedema animal models in an ongoing process to recapitulate the hallmark features of acquired lymphedema in humans, ${ }^{6,24,25}$ we implemented a VLNT animal model performing, to the best of our knowledge, the first contralateral VLNT mouse model. The 3-month follow-up eradicate the possibility that this results can be the consequence of a 


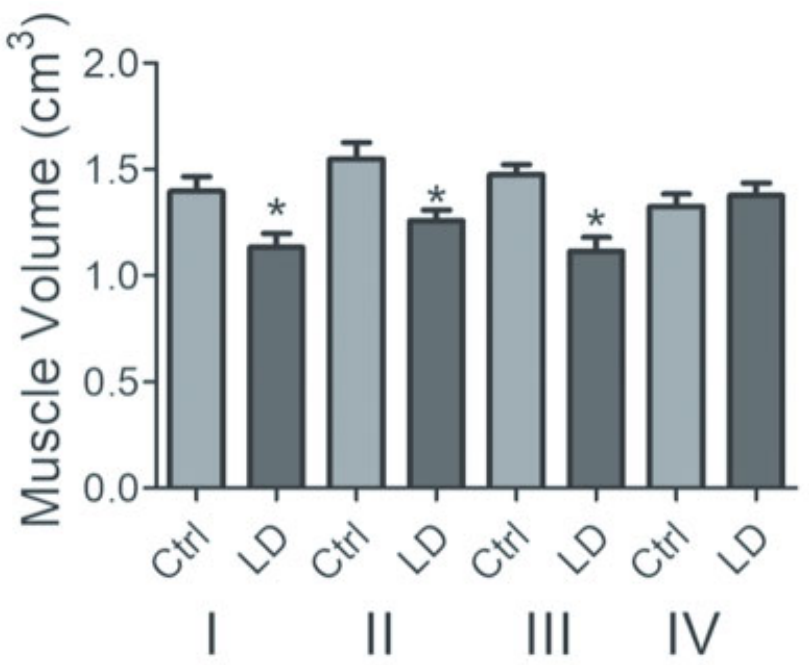

Fig. 17 Muscle volume evaluated by magnetic resonance imaging (MRI) measurement at day 67 after the lymphedema induction. Group 4 showed no skeletal muscle mass loss in comparison to the other three groups $\left({ }^{*} p<0.05\right)$.

spontaneous lymphedema reabsorption, which is one the limitations of rodent lymphedema models. ${ }^{26}$

The purpose of this mouse model is not to exactly reproduce the condition of human VLNT, but to provide a more precise VLNT mouse model than the ones previously described. Although this model cannot reproduce the ischemia/reperfusion injury of a free vascular transfer, which is almost always the technique applied in humans, it can improve the previously described mouse models adding some of the keys of the surgical procedure actually performed in clinics, as the transferred lymph node is vascularized (albeit pediculate), harvested from healthy tissue, and far from the recipient site.

Since the combination of VLNT and VEGF-C had already been described in the literature, ${ }^{27-30}$ we took advantage of the promoting effect of VEGF-C in the VLNT effectiveness to determine whether and how the presence of a lymphangiogenic factor is essential in the VLNT success, after removing the bias of a previously damaged lymph node. In all the analyses performed in this study, the mice group benefiting from the association of VLNT and local VEGF-C administration was significantly detached from the three others groups and displayed a dramatic reduction of lymphedema both at clinical, functional, and histological levels during the 3 months of observation by promoting lymphatic growth/survival, lymphatic fluid drainage efficiency, and reducing skin inflammation. These differences were clear-cut even in comparison to the VLNT from a pristine donor site, which better replicate the procedure actually performed in humans than the ipsilateral VLNT mouse model described so far.

This study shows new interesting data about the presence of skeletal muscle atrophy in association with the development of lymphedema, demonstrated by the MRI analysis. This is a new finding in mice, never reported in the literature, which could benefit from further studies to confirm this skeletal muscle modification and its pathological mechanism of induction and regression.
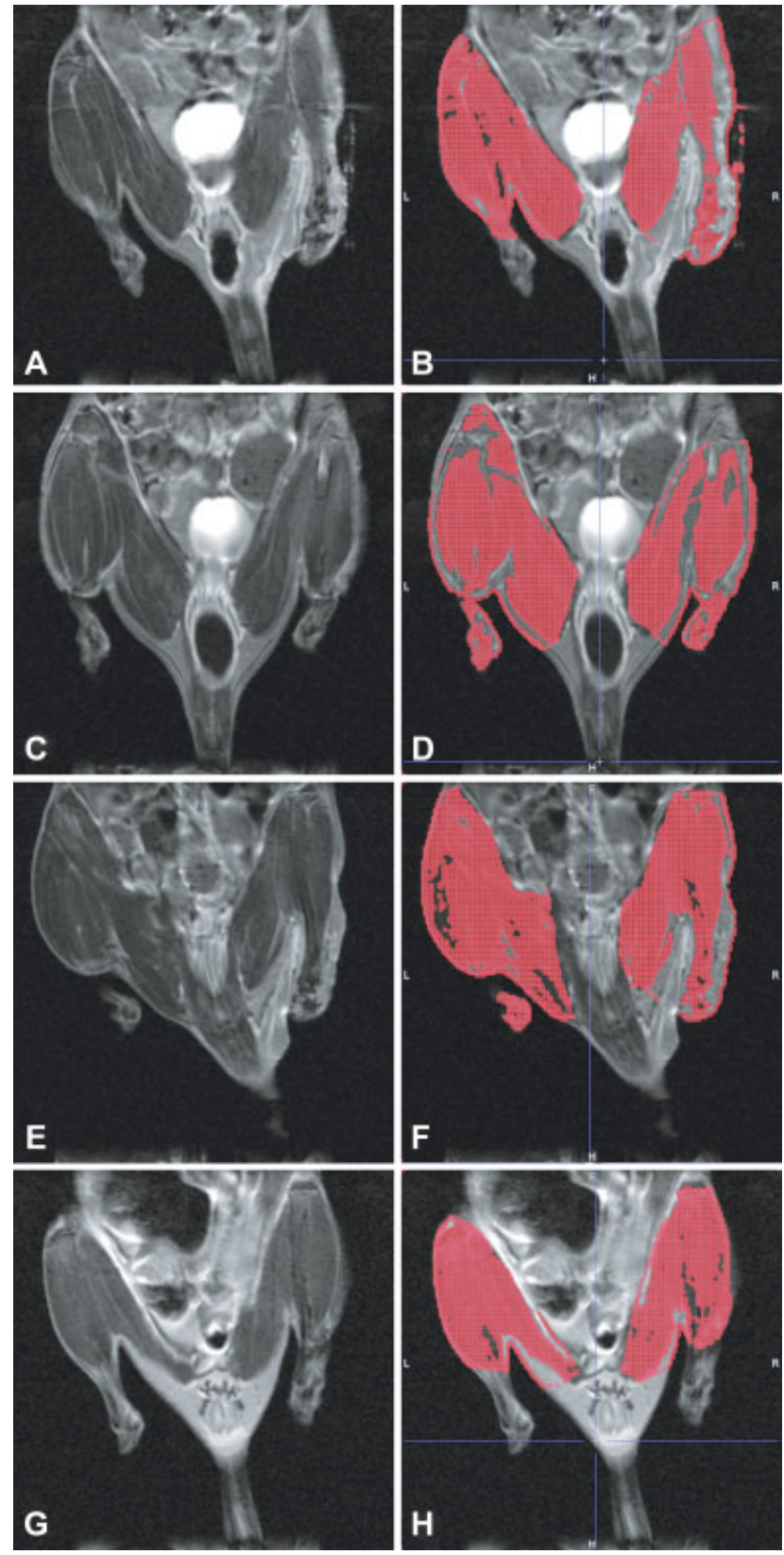

Fig. 18 Magnetic resonance imaging (MRI) images of the four groups. Group $1(\mathrm{~A})$, group $2(\mathrm{C})$, group $3(\mathrm{E})$, and group $4(\mathrm{G})$ paw volume and skeletal muscle mass in the MRI images. The measurements show the absence of skeletal muscle atrophy in group $4(G)$. The red mask represents the segmented muscle for group 1 (B), group 2 (D), group $3(\mathrm{~F})$, and group $4(\mathrm{H})$.

\section{Conclusion}

Currently, the association of VLNT with local prolymphangiogenic factor seems to be the better strategy to improve the clinical outcome of secondary lymphedema. ${ }^{29,31}$ The translation of these interesting data generated in preclinical model to humans will depend on the results of ongoing clinical studies, ${ }^{32}$ which are testing the human safety and efficacy of local VEGF-C administration in enhancing the VLNT in breast cancer-related lymphedema. The VLNT mouse model described in this study provides an effective 
and more precise mouse model for further experimental research about the VLNT impact on lymphedema and confirms the synergetic effect between a lymphangiogenic factor treatment and the lymph node transfer. Additionally, another interest of this model relies on the use of gelatin sponges to locally deliver the VEGF-C: as they are suitable to encapsulate a wide range of biological compounds, this model can thus be used to screen the potential of a molecule in a preclinical model of VLNT.

\section{Conflict of Interest}

None declared.

\section{References}

1 Bergan J, Bunke N. General considerations. In: Lee BB, Bergan J, Rockson SG, eds. Lymphedema. A Concise Compendium of Theory and Practice. London: Springer-Verlag; 2011:3-5

2 Koshima I, Narushima M, Yamamoto Y, Mihara M, Iida T. Recent advancement on surgical treatments for lymphedema. Ann Vasc Dis 2012;5(04):409-415

3 Cheng $\mathrm{MH}$, Huang JJ, Wu CW, et al. The mechanism of vascularized lymph node transfer for lymphedema: natural lymphaticovenous drainage. Plast Reconstr Surg 2014;133(02):192e-198e

4 Scaglioni MF, Arvanitakis M, Chen YC, Giovanoli P, Chia-Shen Yang J, Chang EI. Comprehensive review of vascularized lymph node transfers for lymphedema: Outcomes and complications. Microsurgery 2018;38(02):222-229

5 Frueh FS, Gousopoulos E, Rezaeian F, Menger MD, Lindenblatt N, Giovanoli P. Animal models in surgical lymphedema research-a systematic review. J Surg Res 2016;200(01):208-220

6 Jørgensen MG, Toyserkani NM, Hansen CR, et al. Quantification of chronic lymphedema in a revised mouse model. Ann Plast Surg 2018;81(05):594-603

7 Najjar M, Lopez MM Jr, Ballestin A, et al. Reestablishment of lymphatic drainage after vascularized lymph node transfer in a rat model. Plast Reconstr Surg 2018;142(04):503e-508e

8 Cornelissen AJ, Qiu SS, Lopez Penha T, et al. Outcomes of vascularized versus non-vascularized lymph node transplant in animal models for lymphedema. Review of the literature. J Surg Oncol 2017;115(01):32-36

9 Hayashida K, Yoshida S, Yoshimoto H, et al. Adipose-derived stem cells and vascularized lymph node transfers successfully treat mouse hindlimb secondary lymphedema by early reconnection of the lymphatic system and lymphangiogenesis. Plast Reconstr Surg 2017;139(03):639-651

10 Ishikawa K, Funayama E, Maeda T, et al. Changes in high endothelial venules in lymph nodes after vascularized and nonvascularized lymph node transfer in a murine autograft model. J Surg Oncol 2019;119(06):700-707

11 Ishikawa K, Maeda T, Funayama E, et al. Feasibility of pedicled vascularized inguinal lymph node transfer in a mouse model: a preliminary study. Microsurgery 2019;39(03):247-254

12 Avraham T, Yan A, Zampell JC, et al. Radiation therapy causes loss of dermal lymphatic vessels and interferes with lymphatic function by TGF- $\beta 1$-mediated tissue fibrosis. Am J Physiol Cell Physiol 2010;299(03):C589-C605

13 Harrell MI, Iritani BM, Ruddell A. Lymph node mapping in the mouse. J Immunol Methods 2008;332(1-2):170-174
14 Oashi K, Furukawa H, Oyama A, et al. A new model of acquired lymphedema in the mouse hind limb: a preliminary report. Ann Plast Surg 2012;69(05):565-568

15 Jeltsch M, Kaipainen A, Joukov V, et al. Hyperplasia of lymphatic vessels in VEGF-C transgenic mice. Science 1997;276 (5317):1423-1425

16 Morfoisse F, Renaud E, Hantelys F, Prats AC, Garmy-Susini B. Role of hypoxia and vascular endothelial growth factors in lymphangiogenesis. Mol Cell Oncol 2015;2(04):e1024821

17 Van de Velde M, García-Caballero M, Durré T, Kridelka F, Noël A. Ear sponge assay: a method to investigate angiogenesis and lymphangiogenesis in mice. Methods Mol Biol 2018; 1731:223-233

18 Kapur JN, Sahoo PK, Wong AKC. A new method for grey-level picture theresholding using the entropy of the histogram. Comput Image Process. 1985;29:273-285

19 Tammela T, Alitalo K. Lymphangiogenesis: molecular mechanisms and future promise. Cell 2010;140(04):460-476

20 Ly CL, Kataru RP, Mehrara BJ. Inflammatory manifestations of lymphedema. Int J Mol Sci 2017;18(01):E171

21 Petrek JA, Pressman PI, Smith RA. Lymphedema: current issues in research and management. CA Cancer J Clin 2000;50(05): 292-307, quiz 308-311

22 Boris M, Weindorf S, Lasinkski S. Persistence of lymphedema reduction after noninvasive complex lymphedema therapy. Oncology (Williston Park) 1997;11(01):99-109, discussion 110, 113-114

23 Saaristo AM, Niemi TS, Viitanen TP, Tervala TV, Hartiala P, Suominen EA. Microvascular breast reconstruction and lymph node transfer for postmastectomy lymphedema patients. Ann Surg 2012;255(03):468-473

24 Hadamitzky C, Pabst R. Acquired lymphedema: an urgent need for adequate animal models. Cancer Res 2008;68(02):343-345

25 Iwasaki D, Yamamoto Y, Murao N, Oyama A, Funayama E, Furukawa $\mathrm{H}$. Establishment of an acquired lymphedema model in the mouse hindlimb: technical refinement and molecular characteristics. Plast Reconstr Surg 2017;139(01):67e-78e

26 Ghanta S, Cuzzone DA, Mehrara BJ. Animal models of lymphedema. In: Neligan PC, Masia J, Piller N, eds. Lymphedema. Complete Medical and Surgical Management. Thieme Verlags gruppe; 2016:583-585

27 Tammela T, Saaristo A, Holopainen T, et al. Therapeutic differentiation and maturation of lymphatic vessels after lymph node dissection and transplantation. Nat Med 2007;13(12):1458-1466

28 Hartiala P, Saaristo AM. Growth factor therapy and autologous lymph node transfer in lymphedema. Trends Cardiovasc Med 2010;20(08):249-253

29 Lähteenvuo M, Honkonen K, Tervala T, et al. Growth factor therapy and autologous lymph node transfer in lymphedema. Circulation 2011;123(06):613-620

30 Holopainen T, Bry M, Alitalo K, Saaristo A. Perspectives on lymphangiogenesis and angiogenesis in cancer. J Surg Oncol 2011;103(06):484-488

31 Visuri MT, Honkonen KM, Hartiala P, et al. VEGF-C and VEGFC156S in the pro-lymphangiogenic growth factor therapy of lymphedema: a large animal study. Angiogenesis 2015;18(03): 313-326

32 ClinicalTrials.gov. A Phase I Study With Lymfactin ${ }^{\circledR}$ in the Treatment of Patients With Secondary Lymphedema. Available at: https://clinicaltrials.gov/ct2/show/NCT02994771. Accessed March 6,2019 